\title{
ON THE DEDUCTION OF WIEN'S DISPLACEMENT LAW
}

By E. Buckingham

I. Although Wien's displacement law may be regarded as quite well established by experiment, its great importance seems to justify attempts to improve or simplify the reasoning by which it may be deduced a priori as a consequence of the general principles of thermodynamics and the electromagnetic theory of radiation. Any such deduction must, in substance, contain the following four elements:

(a) The treatment, by Doppler's principle, of the change of wave length produced when diffuse radiation is compressed or expanded within a perfectly reflecting shell, i. e., adiabatically.

(b) The evaluation, by means of the principle of the conservation of energy, of the change of the volume density of the radiant energy which occurs during the adiabatic change of volume and accompanies the change of wave length. This step involves the use of the value of the pressure of diffuse radiation on a bounding surface, deduced from the electromagnetic theory and confirmed by experiment.

(c) The demonstration, by means of the second law of thermodynamics, that black radiation remains black when its density and temperature are changed by adiabatic change of volume.

(d) The use of the Stefan-Boltzmann law to correllate the results obtained by the steps $(a),(b)$, and $(c)$ so that the displacement law shall appear as a necessary consequence of those results. 
These parts of the deduction need not be kept entirely separate nor do they necessarily occur in the order given above, which is that followed in this paper, but they must be present in some form or other. In the deductions I have read, the treatment of the change of wave length seems somewhat difficult or obscure and I have attempted to simplify this part of the subject and make it easier to grasp. The remainder of the demonstration contains little that is at all novel, but is given for the sake of presenting a connected whole, comprehensible to those who are not already familiar with the subject. The treatment is elementary and relates only to radiation in vacuo.

2. Let $d s$ be an infinitesimal plane element of surface at a point within a field of radiation. A certain amount of radiant energy passes through $d s$ from the negative to the positive side, in unit time, in various directions. Let us consider only those directions comprised within a cone of the infinitesimal solid angle $d w$ described about the positive normal to $d s$. The amount of energy of wave lengths between $\lambda$ and $\lambda+d \lambda$, which passes through $d s$ from the negative to the positive side in one second, in directions close enough to the normal to lie within the cone, may be expressed by

$$
R_{\lambda} d \lambda \cdot d s \cdot d w
$$

The quantity $R_{\lambda} d \lambda$ may be called the strength of the radiation and $R_{\lambda}$ the "radiant vector" at the given point, in the given direction of the positive normal to $d s$, and for the wave length $\lambda$. If the value of $R_{\lambda}$ is given for all values of $\lambda$, for all directions, and at every point within a given region, the radiation within that region is, for our purposes, completely specified, since questions of phase and state of polarization will not enter into our reasoning.

By speaking of "the energy of wave lengths between $\lambda$ and $\lambda+d \lambda$ " we make a somewhat violent though familiar assumption, namely, that no matter what may be the nature of the pulses which constitute radiation, since our spectral apparatus enables us to analyze radiation into series of wave trains of assignable period, the radiation before analysis may be treated as the sum of these wave trains coexisting independently. However obvious the 
truth of this assumption may appear from a purely mathematical standpoint, it is well to recognize that physically it is to be justified by the agreement with experiment of conclusions drawn from it. It is in fact thus justified and we shall make a rather full use of this principle.

3. Let us consider a closed evacuated shell, the walls of which reflect perfectly, but at least somewhat irregularly. Let a beam of approximately monochromatic radiation of wave lengths between $\lambda$ and $\lambda+d \lambda$ be admitted to the inclosure through a hole, which is then closed by a cover similar in its properties to the rest of the walls. After a short time the radiation within the shell becomes perfectly diffuse, for the directed quality of the original beam is soon obliterated by the successive irregular reflexions, so that thereafter the value of $R_{\lambda}$ is the same at all points and in all directions. These reflexions, however, do not change the period of the radiation, since there is no absorption and reemission, but only pure reflexion. The volume density of the energy is now

$$
\rho_{\lambda} d \lambda=4 \pi \frac{R_{\lambda} d \lambda}{c}
$$

where $c$ is the velocity of light; and the whole amount of energy within the shell is $v \rho_{\lambda} d \lambda$, if $v$ is the volume of the shell.

Let $M$ be a small plane piece of the shell wall of area $s$, and let $M$ be given a normal velocity $\beta c$ outward, $\beta$ being infinitesimal. This motion will disturb the perfect diffuseness of $R_{\lambda}$ by an amount which we shall show to be negligible, but at present we shall assume that $R_{\lambda}$ remains diffuse.

The reflexion from $M$ also causes a change of period which we must now proceed to evaluate. If a wave train of period $T$ strikes $M$ at an angle of incidence $\varphi$, it is easily seen that the period $T^{\prime}$ of arrival of the waves at a given point of $M$ is

$$
T^{\prime}=\frac{T}{\mathrm{I}-\beta \cos \phi}
$$

The period at a point of the moving surface is therefore greater than at a fixed point in space in the ratio

$$
r_{a}=\mathrm{I}+\beta \cos \phi
$$


terms of higher orders in $\beta$ being negligible. A disturbance starting with the period $T^{\prime}$ at a point of the moving surface and propagated at an angle $\psi$ with the normal has, upon arrival at a fixed point in space, the period

$$
T^{\prime \prime}=T^{\prime}(\mathrm{I}+\beta \cos \psi)
$$

so that the effect of departure is to increase the period in the ratio

$$
r_{d}=\mathrm{I}+\beta \cos \psi
$$

Our problem is to find the total effect on the original period $T$, of all the arrivals and departures, at all possible angles $\varphi$ and $\psi$ from $o$ to $\pi / 2$, during a long time $t$; and to do this we must find the number of times that each of these effects is produced on every wave; i. e., every element of the radiation existing within the shell.

4. We base our reasoning on the consideration that in a very long time-though not in a short one-every element of the energy within the shell must undergo reflexion from $M$ at the angles $(\phi, \psi)$ just as many times as every other element. The number of times that any particular effect of reflexion at $M$ is produced on each element of the energy is therefore the ratio of the total amount of energy thus affected in the time $t$ to the total amount present within the shell. The changes of period in the ratios $r_{a}$ and $r_{d}$, caused by arrival and departure, occur alternately; and in finding the total effect of a number of successive arrivals and departures we have evidently to evaluate a product of the form

$$
r_{a} r_{d} \cdot r_{a}^{\prime}{ }_{a}{ }^{\prime}{ }_{d} \cdot r^{\prime \prime}{ }_{a} r^{\prime \prime}{ }_{d} \text {, etc. }
$$

But since multiplication is commutative, we shall get the same result if we pursue the more convenient method of treating all the arrivals by themselves, then all the departures by themselves, and finally multiplying the two resulting ratios together to get the combined effect of both arrivals and departures.

We start, then, with the arrivals. In any time $t$, the amount of energy which strikes $M$ at angles between $\varphi$ and $\varphi+d \varphi$ is

$$
t \cdot R_{\lambda} d \lambda \mathrm{s} \cos \varphi \cdot 2 \pi \sin \varphi d \varphi
$$


The total amount present within the shell to be affected is, by equation (I),

$$
v \cdot \frac{4 \pi R_{\lambda} d \lambda}{c}
$$

Hence the number of times $n$ that each element of the energy arrives at $M$ at angles between $\phi$ and $\phi+d \phi$ within the long time $t$ is

$$
\begin{aligned}
n & =\frac{t \cdot R_{\lambda} d \lambda s \cos \varphi \cdot 2 \pi \sin \varphi d \phi}{v \cdot \frac{4 \pi R_{\lambda} d \lambda}{c}} \\
& =\frac{c t s}{2 v} \cos \phi \sin \varphi d \phi
\end{aligned}
$$

By equation (2) the effect of all these $n$ arrivals is to increase the period in the ratio

$$
r_{a}^{n}=(\mathrm{I}+\beta \cos \varphi)^{n}=\mathrm{I}+n \beta \cos \varphi
$$

the remaining terms being of higher orders in $\beta$. Inserting the value of $n$ from (4) we have

$$
r_{a}^{n}=\mathrm{I}+\frac{\beta c t s}{2 v} \cos ^{2} \phi \sin \varphi d \varphi
$$

and since $\beta c t$ is the infinitesimal increase of volume, $\Delta v$, which occurs within the time $t$ as a result of the motion of $M$, this last equation may be written in the form

$$
r_{a}^{n}=\mathrm{I}+\frac{\mathrm{I}}{2} \frac{\Delta v}{v} \cos ^{2} \phi \sin \phi d \phi
$$

So far we have considered only the directions between $\phi$ and $\varphi+d \phi$; but meanwhile the given element of energy has also been arriving a large number of times at every other possible angle between $o$ and $\pi / 2$, and equation (6), with the appropriate value of $\varphi$, is applicable to every such angle. The total effect of all the arrivals at all possible angles will therefore be to change the period in the ratio given by the product of all the expressions of the form (6) for all values of $\phi$; and dropping terms of higher order in $\beta$, the value of this product will be 


$$
\mathrm{I}+\frac{\mathrm{I}}{2} \frac{\Delta v}{v} \int_{0}^{\frac{\pi}{2}} \cos ^{2} \phi \sin \varphi d \varphi=\mathrm{I}+\frac{\mathrm{I}}{6} \frac{\Delta v}{v}
$$

If we go on to treat the effects of the departures, the reasoning will be found to be the same all the way through, with the mere substitution of $\psi$ for $\varphi$, and the total effect will be found as before to be to increase the period in the ratio $\mathrm{I}+\frac{\mathrm{I}}{6} \frac{\Delta v}{v}$. Hence the combined result of the two sets of effects, which have in reality been occurring alternately, is to increase the period of every element of the radiation in the ratio

$$
\frac{T+\Delta T}{T}=\left(\mathrm{I}+\frac{\mathrm{I}}{6} \frac{\Delta v}{v}\right)^{2}=\mathrm{I}+\frac{\mathrm{I}}{3} \frac{\Delta v}{v}
$$

Replacing the period by the wave length we therefore have

$$
\frac{\Delta \lambda}{\lambda}=\frac{\mathrm{I}}{3} \frac{\Delta v}{v}
$$

Since this result is independent of the original value of the wavelength $\lambda$, it is valid for any value. Hence $\lambda+d \lambda$, and therefore $d \lambda$, the interval within which the wave-lengths are included, is changed in the same ratio as $\lambda$.

The result is also valid for any element of the surface of the shell which is small enough to be treated as plane, and for motion either in or out, so that equation (9) may be integrated into the form

$$
\lambda=\text { const. } \times \sqrt[3]{v}
$$

The meaning of equation (го), reduced to its simplest terms, is as follows: If the shell changes its volume while retaining its shape, the dimensions of the waves change in the same ratio as those of the shell. The whole system of waves and shell remains geometrically similar to itself, the number of waves present being unchanged.

5. In the foregoing reasoning we have treated the radiation as perfectly diffuse, for the cancellation of $R_{\lambda}$ from the numerator 
and denominator of equation (4) and the corresponding equation for the case of departure, involved the assumption that $R_{\lambda}$ was the same at all points and in all directions. This assumption is not exact, for upon reflection from a moving mirror the angle of departure $\psi^{\prime}$ differs from the angle $\psi$ at which the ray would leave a fixed mirror by a quantity of the order of magnitude of the ratio of the velocity of the mirror to the velocity of light. The result of this is that at any point within our shell the radiant vector in directions away from the moving piece $M$ has not exactly the mean value $R_{\lambda}$, but a value $R_{\lambda}{ }^{\prime}=R_{\lambda}(\mathrm{I}+\epsilon)$, where $\epsilon$ is an infinitesimal of the same order as $\beta$. This departure from perfect diffuseness is not cumulative, but remains of the same order of magnitude whatever the lapse of time, for the disturbing effect of reflection from $M$ is continually being damped out by the diffusing effect of the irregular reflections from the stationary walls.

If we now review our reasoning, we find that $R_{\lambda}$ appears only in the expressions for the total energy within the shell and for the total energy which strikes or leaves $M$ at a given angle within the time $t$. If $R_{\lambda}$ represents the average value which satisfies the equation

$$
\int \rho_{\lambda} d \lambda \cdot d v=4 \pi \frac{R_{\lambda} d \lambda}{c} \cdot v
$$

the numerator of equation (4) ought to contain not $R_{\lambda}$ but $R_{\lambda}(\mathrm{I}+\eta)$, where $\eta$ is an infinitesimal of the order $\beta q, q$ being the ratio of the area $s$ to the whole area of the shell walls. The error in $n$ caused by the omission of this factor $(\mathrm{I}+\eta)$ is infinitesimal and negligible. The changes caused in equations (5) to (Io) by using the exact value instead of the mean value of $R_{\lambda}$ would all be of a lower order of magnitude than the terms which have been retained. Hence the error incurred by our treating the radiation as completely diffuse is infinitesimal, and the result expressed in equations (9) and (Io) remains valid.

One further point may be worth notice. If " the long time $t$ " appears to the reader to be possibly not long enough to give all the finite amount of energy within the shell an equal opportunity of being reflected from $M$ in every one of the infinite number of conceivable ways, there is no objection to his making it longer, in 
other words, infinite. If this is done, we may still make $\Delta v$ or ct $\beta s$ infinitesimal, as we want it to be, by making $\beta s$ of the order $\frac{I}{t^{2}}$; and this may be accomplished either by making $\beta=\frac{\text { const }}{t^{2}}$ with $s$ finite, or by making both $\beta$ and $s$ of the order $\frac{\mathrm{I}}{t}$. There is nothing to prevent our adopting either course. The use of the more concrete expression a "long" time instead of an "infinite" time did not, therefore, involve an error in the reasoning, while it obviated the necessity of interrupting the argument at an inconvenient point.

6. We have next to consider the change of the energy of the radiation which accompanies its change of period, and we assume that diffuse radiation exerts a pressure equal to one-third of its density on the walls of a containing envelope. This proposition may be deduced in several ways from Maxwell's theory of the electromagnetic field, which has been confirmed in so many respects that we need not regard it as doubtful but accept its consequences without further discussion.

During an expansion $\Delta v$, the work given out is then expressed by $\frac{\mathrm{I}}{3} \rho_{\lambda} d \lambda \cdot \Delta v$; and since the expansion of radiation within a perfect reflector is adiabatic, this work is equal to the simultaneous decrease of the energy within the shell, and we have

$$
\frac{\mathrm{I}}{3} \rho_{\lambda} d \lambda \cdot \Delta v=-\Delta\left(\rho_{\lambda} d \lambda \cdot v\right)
$$

whence upon developing, rearranging, and dividing by $\rho_{\lambda} d \lambda \cdot v$, we have

$$
\frac{4}{3} \frac{\Delta v}{v}+\frac{\Delta d \lambda}{d \lambda}+\frac{\Delta \rho_{\lambda}}{\rho_{\lambda}}=0
$$

But since $d \lambda$ changes in the same ratio as $\lambda, \frac{\Delta d \lambda}{d \lambda}=\frac{\Delta \lambda}{\lambda}$, and if we eliminate $v$ by equation (9) we have

or

$$
5 \frac{\Delta \lambda}{\lambda}+\frac{\Delta \rho_{\lambda}}{\rho_{\lambda}}=\mathrm{C}
$$

$$
\rho_{\lambda} \lambda^{5}=\text { const. }
$$


The meaning of this result is that if approximately monochromatic diffuse radiation of strength $R_{\lambda} d \lambda$ and density $\rho_{\lambda} d \lambda$ is compressed or expanded adiabatically and infinitely slowly, the quantity $\rho_{\lambda}$ changes as the inverse $5^{\text {th }}$ power of the wave length, $\lambda$ itself being subject to equation (Io). If the strip $A$ (Fig. I), of width $d \lambda$ and height $\rho_{\lambda}$, represents the original energy density, the strip $B$ which represents the energy density after compression, has its height increased in the ratio of the $5^{\text {th }}$ power of the ratio of decrease of the width or of mean wave length. Its area is therefore proportional to the inverse $4^{\text {th }}$ power of the wave length.

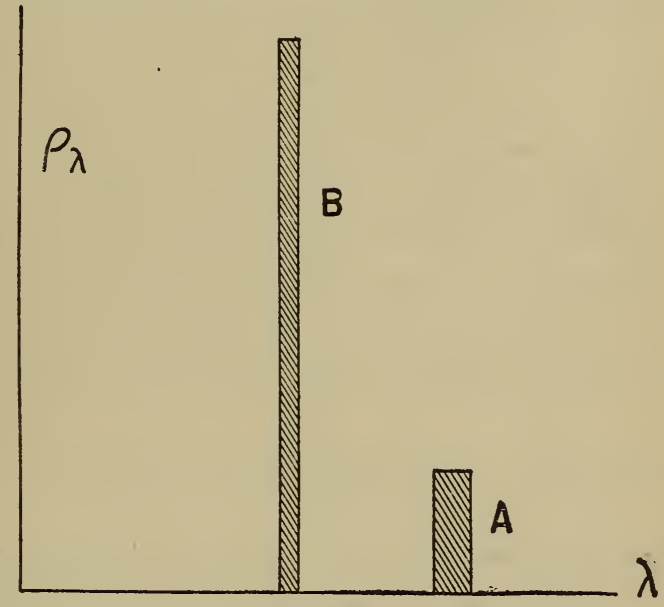

Fig. 1

This change of density and the accompanying change of $R_{\lambda} d \lambda$ do not interfere with the validity of the reasoning by which we found the value of $n$ in equation (4). For since the motion is infinitely slow, the values of $R_{\lambda} d \lambda$ which have been canceled from the numerator and denominator may be treated as always equal, within an infinitesimal amount, in spite of the fact that they are not constant in time.

The width $d \lambda$ may be as small as we please, so that the change of $\rho_{\lambda}$ may be assigned definitely to the particular wave length $\lambda$. There is no occasion for the formation of any new waves; the change in energy is a change in the amplitude of waves already present, which occurs in connection with the change of period $56 \mathrm{IO}^{\circ}-\mathrm{I} 2-8$ 
upon reflection from the moving surface. In a short time, reflection from the moving mirror would introduce inhomogeneity into the radiation, which would not have time to be all equally affected by reflection at $M$; but in a long time, the radiation again becomes homogeneous to the same degree as at first, and equation (12) is satisfied for each wave length.

7. Hitherto we have considered only a small interval of wave lengths, but suppose that the strip in question is merely a part of a continuous distribution of diffuse radiation which may be represented by a curve such as is shown in figure 2. If we take full advantage of the principle of the independence of the separate

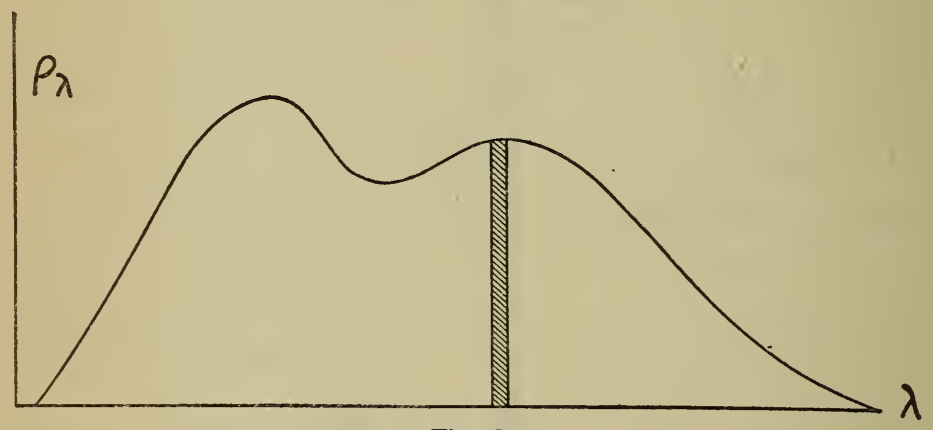

Fig. 2

elements composing the whole spectrum, we must admit that the reasoning given above for an isolated strip is applicable without change to every wave length of the spectrum, no matter what may be the form of the energy curve $\rho_{\lambda}=f(\lambda)$. We then have the proposition that when any completely diffuse radiation is compressed infinitely slowly within a perfectly reflecting inclosure, the energy curve is changed in such a way that the abscissa of every point is multiplied by some fraction $f$ while the ordinate is multiplied by $f^{-5}$, so that the area under the curve, or the integral density of the energy present, is multiplied by $f^{-4}$. This is true for any continuous or discontinuous spectral distribution and not merely for black radiation.

8. The remainder of the deduction contains nothing new, but may be given for the sake of completeness. Let the shell be 
filled with black radiation of temperature $\theta$ and density $\rho$, by first covering a hole in the shell with an ordinary body of temperature $\theta$, and then, after equilibrium has been established, closing the hole with a cover which has the same reflecting properties as the rest of the shell wall. Let the volume of the shell, which may, if we prefer, have the form of a cylinder closed by a piston, be decreased a finite amount by an infinitely slow compression. The fact that this requires an infinite time need not concern us. The density of the energy is increased on account both of the work done and of the decrease of volume of the energy already present. The spectral distribution also changes in the manner already given.

At the end of the compression we introduce into the shell a particle of ordinary matțer so small as to be of negligible thermal capacity. If the spectral distribution after compression was not that of black radiation of the same integral density, absorption and reemission by the particle cause a redistribution and a "blackening" of the radiation without change of density. This establishment of stable equilibrium by redistribution of the energy among the different periods is spontaneous and therefore irreversible.

We now expand to the original volume. The radiation remains black on account of the presence of the particle, and the work given out is the same as that put in during the compression, because the pressure depends only on the total density of the energy present and not on its spectral distribution. At the end of the cycle, which may be completed by removing the particle, we have therefore reestablished the original state exactly. No heat has been added to or taken from any outside body, the work done has been regained, and no changes remain. The cycle is therefore reversible and can not have included any irreversible element. Hence the introduction of the particle after compression did not cause any change in the spectral distribution of the energy which must therefore already have been that of radiation from a black body. Hence we conclude that during infinitely slow adiabatic change of density, radiation which was initially black remains black. 
9. We may now apply equation (I2) to an adiabatic change of the volume and density of black radiation. The integral density changes from that needed for equilibrium with an absorbing shell at the absolute temperature $\theta_{1}$ to that needed for some other temperature $\theta_{2}$. The abscissa $\lambda_{1}$ of any point on the original energy curve is changed to a new value $\lambda_{2}$, such that

$$
\frac{\lambda_{2}}{\lambda_{1}}=x=\sqrt[3]{\frac{v_{2}}{v_{1}}}
$$

At the same time the ordinate of the point changes so that

$$
\frac{\left(\rho_{\lambda}\right)_{2}}{\left(\rho_{\lambda}\right)_{1}}=\frac{I}{x^{5}}
$$

and the area under the curve, which represents the integral density $\rho$, changes so that

$$
\frac{\rho_{2}}{\rho_{1}}=\frac{\mathrm{I}}{x^{4}}
$$

But we know by the Stefan-Boltzmann law that for diffuse black radiation at $\theta_{1}$ and $\theta_{2}$

$$
\frac{\rho_{2}}{\rho_{1}}=\left(\frac{\theta_{2}}{\theta_{1}}\right)^{4}
$$

whence it follows that

$$
x=\frac{\theta_{1}}{\theta_{2}}
$$

From equations ( I $_{3}$ ) and ( 17 ) we thus obtain theorelation

$$
\lambda_{1} \theta_{1}=\lambda_{2} \theta_{2}
$$

and the displacement law contained in equations (I2) and (18) may be stated as follows: Given the spectral energy curve of black radiation at any temperature $\theta_{1}$, to construct the curve for any other temperature $\theta_{2}$, multiply the abscissa of each point by $\frac{\theta_{1}}{\theta_{2}}$ and the coordinate by $\left(\frac{\theta_{2}}{\theta_{1}}\right)^{5}$. "Corresponding" points on the two curves have the same value of $\lambda \theta$. 
From this we may easily deduce the more familiar special forms of the displacement law

$$
\begin{gathered}
\lambda_{\max } \theta=\text { const, } \\
\rho_{\max } \lambda^{5}{ }_{\text {max }}=\text { const, }
\end{gathered}
$$

and

$$
\rho_{\max }=\operatorname{const} \times \theta^{5},
$$

as well as the fact that if the displacement and Stefan-Boltzmann laws are to be satisfied, the complete equation must have the general form

$$
\rho_{\lambda, \theta}=C \lambda-5 F(\lambda \theta)
$$

The only novelties in the above deduction, if there are any at all, occur in sections 4 to 7 ; but it seems to me that greater clearness has been attained without any real sacrifice of rigor and with only very elementary reasoning.

WASHINGTON, February 28, I912. 